\title{
Fatores de insucesso do tratamento cirúrgico da síndrome do túnel do carpo: Quando e como revisar a cirurgia de descompressão do túnel do carpo
}

\section{Failure factors for carpal tunnel syndrome surgical treatment: When and how to perform a revision carpal tunnel decompression surgery}

\author{
Carlos Henrique Fernandes ${ }^{1}$ João Baptista Gomes dos Santos ${ }^{1}{ }^{\circledR}$ Francisco Schwartz-Fernandes ${ }^{2}$ )
} A. Lee Ostermann ${ }^{3(0)}$ Flávio Faloppa10

\footnotetext{
${ }^{1}$ Departamento de Ortopedia e Traumatologia, Escola Paulista de Medicina, Universidade Federal de São Paulo, São Paulo, SP, Brasil

2 USF Health Morsani College of Medicine, Tampa, Flórida, United States

${ }^{3}$ Hand and Orthopaedic Surgery Jefferson Medical College, Thomas Jefferson University, Philadelphia, Pennsylvania, United States
}

Endereço para correspondência Carlos Henrique Fernandes, MD, PhD, Avenida Leôncio de Magalhães, 1021, São Paulo, SP, Brasil (e-mail: carloshandsurgery@gmail.com).

Rev Bras Ortop 2022;57(5):718-725.

\section{Resumo \\ Palavras-chave \\ - síndrome do túnel do carpo/diagnóstico \\ - síndrome do túnel do carpo/cirurgia \\ - síndrome do túnel do carpo/complicações \\ - recidiva \\ - retalhos cirúrgicos}

\section{Abstract}

Apesar de ser um procedimento amplamente utilizado em todo o mundo e com elevadas taxas de remissão dos sintomas, o tratamento cirúrgico da síndrome do túnel do carpo pode apresentar resultados não satisfatórios ao paciente. Esse resultado não satisfatório pode se manifestar clinicamente pela não remissão dos sintomas, remissão dos sintomas mas recorrência desses após um período de tempo da cirurgia ou aparecimento de diferentes sintomas após a cirurgia. Diferentes fatores estão relacionados a esse insucesso do tratamento cirúrgico da síndrome do túnel do carpo (ITCSTC). A prevenção pode ser conseguida por meio de minuciosa avaliação clínica do paciente no período pré-operatório. Dessa forma o cirurgião poderá fazer diagnósticos diferenciais ou diagnósticos concomitantes, assim como identificar fatores ligados a insatisfação do paciente. Os fatores peroperatórios incluem a correta identificação das estruturas anatômicas para completa descompressão do nervo mediano. Inúmeros procedimentos têm sido descritos para o tratamento dos fatores que ocorrem no período pós-operatório. Desses o mais comum, a formação de aderências em torno do nervo mediano, tem sido tratado com relativo sucesso utilizando diferentes retalhos vascularizados ou cobertura com o uso de tecido autólogo ou homólogo. Descreveremos a abordagem do ITCSTC com maiores detalhes no texto.

Despite being a procedure widely used all over the world with high rates of symptom remission, surgical treatment of carpal tunnel syndrome may present unsatisfactory outcomes. Such outcomes may be manifested clinically by non-remission of symptoms, recebido

20 de Dezembro de 2019

aceito

20 de Abril de 2020

Publicado on-line

Setembro 22, 2020
DOI https://doi.org/

$10.1055 / \mathrm{s}-0040-1713759$. ISSN $0102-3616$ (c) 2020. Sociedade Brasileira de Ortopedia e Traumatologia. All rights reserved.

This is an open access article published by Thieme under the terms of the Creative Commons Attribution-NonDerivative-NonCommercial-License, permitting copying and reproduction so long as the original work is given appropriate credit. Contents may not be used for commercial purposes, or adapted, remixed, transformed or built upon. (https://creativecommons.org/ licenses/by-nc-nd/4.0/)

Thieme Revinter Publicações Ltda., Rua do Matoso 170, Rio de Janeiro, RJ, CEP 20270-135, Brazil 


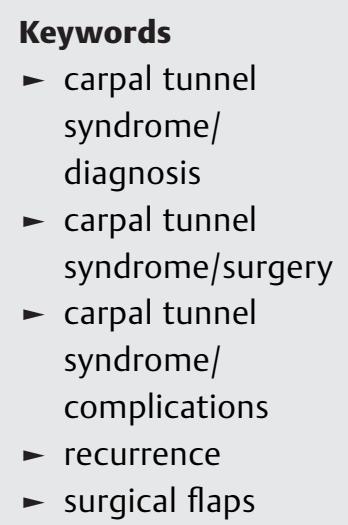

Keywords syndrome/ diagnosis

carpal tunnel

carpal tunnel syndrome/

- recurrence

- surgical flaps

remission of symptoms with recurrence a time after surgery or appearance of different symptoms after surgery. Different factors are related to this unsuccessful surgical treatment of carpal tunnel syndrome. Prevention can be achieved through a thorough preoperative clinical evaluation of the patient. As such, the surgeon will be able to make differential or concomitant diagnoses, as well as determine factors related to patient dissatisfaction. Perioperative factors include the correct identification of anatomical structures for complete median nerve decompression. Numerous procedures have been described for managing postoperative factors. Among them, the most common is adhesion around the median nerve, which has been treated with relative success using different vascularized flaps or autologous or homologous tissue coverage. The approach to cases with unsuccessful surgical treatment of carpal tunnel syndrome is discussed in more detail in the text.

\section{Introdução}

A síndrome do túnel do carpo (STC) ocorre em 3\% a 5\% da população em geral. ${ }^{1}$ Karpitskaya et al. ${ }^{2}$ observaram que a obesidade, hipotireoidismo e diabetes são mais prevalentes em pacientes com STC quando comparados à população geral, mas não encontraram diferenças quanto ao tabagismo. Aproximadamente 2 a 4 em cada 100 adultos, apresentando sintomas de STC, serão submetidos a tratamento cirúrgico. ${ }^{3}$ O tratamento cirúrgico da síndrome do túnel do carpo, que consiste na secção do ligamento transverso do carpo com consequente descompressão do nervo mediano, tem demonstrado ser um tratamento eficaz para remissão dos sintomas dos pacientes. ${ }^{4}$ Embora seja um procedimento realizado com muita frequência, os casos de não satisfação dos pacientes após o tratamento cirúrgico são mais frequentes que o esperado. ${ }^{5}$ Estudos mostram que as recidivas podem ocorrer entre 3\% a 20\% dos casos. ${ }^{6,7}$ Essa situação provoca insatisfação do paciente e frustração para o cirurgião. Nos Estados Unidos, uma ação judicial, após uma cirurgia de síndrome do túnel do carpo, pode custar ao cirurgião o valor entre US \$300.000,00 a US\$ 600.000,00 dólares. $^{8}$

O insucesso do tratamento cirúrgico da síndrome do túnel do carpo (ITCSTC) pode se apresentar como três diferentes situações clínicas quanto aos sintomas. A primeira situação é aquela na qual os pacientes apresentam sintomas persistentes após o procedimento cirúrgico. A segunda situação clínica é aquela na qual após o tratamento cirúrgico há remissão dos sintomas temporariamente e depois de um período de tempo há a recidiva dos sintomas. A terceira situação clínica é a remissão dos sintomas prévios à cirurgia mas os pacientes referem diferentes sintomas no pós-operatórios. ${ }^{9}$ Zieske et al. $^{10}$ reportaram, que os sintomas persistentes são responsáveis por aproximadamente $40 \%$ das queixas em pacientes submetidos à revisão da cirurgia para STC. A piora dos sintomas e paralisia da musculatura tenar após a cirurgia são relacionadas a lesão iatrogênica e variam de $15 \%$ e $67 \%$ das causas de ITCSTC. ${ }^{10-12}$ Nos casos de suspeita de lesão iatrogênica, está indicada uma reintervenção precoce para confirmação diagnóstica e tratamento. ${ }^{5}$
Consideramos que o mais importante é o cirurgião identificar possíveis fatores que poderão provocar o ITCSTC e prevenir sua ocorrência. Uma revisão de cirurgia de síndrome do túnel do carpo deve ser muito bem programada porque até $40 \%$ dos pacientes apresentam desfechos desfavoráveis. ${ }^{10,13}$

Suportada pelos estudos encontrados na literatura, essa revisão reflete a experiência dos autores com o tratamento cirúrgico da síndrome do túnel do carpo em pacientes que não se sentiram satisfeitos após o tratamento inicial.

\section{Fatores de insucesso do tratamento cirúrgico da Síndrome do Túnel do Carpo}

São diversos os fatores que podem levar ao ITCSTC. Podemos dividir em fatores pré-operatórios, per-operatórios e pósoperatórios. Nem sempre é fácil para o cirurgião reconhecer esses fatores. Muitas vezes o diagnóstico e o procedimento cirúrgico são subestimados, considerados simples e fáceis pelos pacientes e cirurgiões.

O primeiro fator a ser considerado é quanto ao diagnóstico pré-operatório, seu diagnóstico diferencial e doenças associadas. Por definição, síndrome é um conjunto de sinais e sintomas que se encontram associados a uma entidade conhecida ou não. A literatura médica é farta em demonstrar uma grande variação de sinais e sintomas nos pacientes com síndrome do túnel do carpo. Em 2009, a American Academy of Orthopaedic Surgeons (AAOS) publicou as recomendações para realização do diagnóstico da STC. Estas recomendações não são absolutas e o tratamento do paciente deve ser baseado sempre no julgamento independente do médico e de acordo com as circunstâncias clínicas individuais. ${ }^{14}$ As recomendações da AAOS incluem que o cirurgião tenha uma detalhada história clinica do paciente, realize o exame físico do paciente, incluindo as características pessoais, testes provocativos, exame da sensibilidade, exame muscular e / ou testes discriminatórios para diagnósticos diferenciais. ${ }^{14}$ Apesar dessas recomendações, o diagnóstico da STC nem sempre é fácil. Não existe uma "receita de bolo" para esse diagnóstico. A apresentação mais comum de STC, é a 
parestesia afetando os dedos radiais da mão como o polegar, indicador e médio, mas a apresentação clínica pode variar de um paciente para outro. ${ }^{15}$ Além da variabilidade de sintomas clínicos, que na grande maioria das vezes são subjetivos, a síndrome pode ocorrer em associação ou concomitância com doenças sistêmicas. Vale lembrar que a sensação de formigamentos na mão, queixa mais frequente nos pacientes com STC, pode ocorrer na população geral, sem síndrome do túnel do carpo. ${ }^{1}$ Avaliação clinica detalhada, inclui a pesquisa dos testes provocativos, o sinal de Tinel, a manobra de Durkan, o teste de Phalen e o "scratch collapse test". ${ }^{16}$ o Sinal de Tinel tem $60 \%$ de sensibilidade e $67 \%$ de especificidade e o teste de Phalen tem $75 \%$ de sensibilidade e $47 \%$ de especificidade. ${ }^{17}$

A avaliação da sensibilidade da mão pode ser feita por diferentes métodos, como o teste de discriminação de dois pontos e o teste com monofilamentos de nylon. ${ }^{18,19} \mathrm{~A}$ combinação do teste de monofilamentos com o teste de flexão do punho de Phalen para o diagnóstico de STC tem sensibilidade de $82 \%$ e especificidade de $86 \% .{ }^{20}$ A limitação dos testes provocativos, de sensibilidade e força, é a subjetividade. As respostas aos testes provocativos, ao estímulo pelos monofilamentos ou à força exercida para realizar a preensão do dinamômetro são determinadas pelo paciente. Não há como o cirurgião saber se o paciente está simulando durante a realização do exame.

O exame de eletroneuromiografia (ENMG), considerado padrão ouro para confirmação diagnóstica, também pode apresentar alterações em pacientes assintomáticos. ${ }^{21} \mathrm{O}$ exame de ENMG deve ser realizado para diagnóstico diferencial, na presença de atrofia tenar e/ou dormência persistente ou nos casos de suspeita clínica e que o tratamento cirúrgico está sendo considerado. Nestes casos o exame deverá ser realizado seguindo o protocolo da American Academy of Neurology (AAN), American Association of Neuromuscular and Electrodiagnostic Medicine (AANEM) e American Academy of Physical Medicine and Rehabilitation (AAPMR). ${ }^{14}$ A graduação da intensidade da compressão em leve, moderada ou grave, é útil pois pacientes com síndrome do túnel do carpo leve ou moderada experimentam um tempo mais rápido para resolução dos sintomas sensitivos quando comparados aos pacientes com síndrome do túnel do carpo grave. ${ }^{22}$ Uma limitação da ENMG é a persistência de alterações elétricas mesmo nos pacientes assintomáticos após a cirurgia, portanto consideramos que a ENMG não é um bom parâmetro para avaliar os resultados de uma descompressão do nervo mediano no túnel do carpo. A exceção ocorre se há piora dos sintomas após um procedimento cirúrgico com suspeita de lesão iatrogênica, onde a ENMG mostrará uma piora dos parâmetros avaliados.

O exame de ultrassonografia pode ser útil para confirmação diagnóstica. Estudo mostra que a área transversal do nervo mediano encontrada no exame pré-operatório, diminui a partir da 4a semana pós-operatória. ${ }^{23}$ Apresenta como desvantagem ser um exame operador dependente.

Outros exames como a ressonância magnética, tomografia axial computadorizada ou potencial evocado não devem ser utilizados de rotina. ${ }^{14}$

Os protocolos de autoavaliação podem ser utilizados para avaliar a gravidade dos sintomas e a função da mão nos pacientes com STC. O questionário criado por Levine, tem sido utilizado em trabalhos internacionais e foi traduzido e validado para o português do Brasil. ${ }^{24}$ A desvantagem do questionário de Levine é ser longo e demorado. O questionário criado por Atroshi, o CTS-6 , é curto, de fácil entendimento pelo paciente e de rápido preenchimento, também foi traduzido para o português do Brasil. ${ }^{25}$ O Brief Michigan Hand Questionnaire também é curto e de fácil aplicação mas é menos específico para a STC. ${ }^{26}$

Em determinadas situações da prática clínica, o diagnóstico acurado pode prevenir a insatisfação do paciente após o tratamento cirúrgico da síndrome do túnel do carpo. ${ }^{20} \mathrm{~A}$ primeira situação é aquela na qual o paciente apresenta sintomas parestésicos na mão, mas a compressão do nervosa não ocorre no no túnel do carpo e sim em outro local. Os diagnósticos diferenciais mais comuns são a síndrome do pronador redondo, a compressão pelo lacertus fibrosus e hérnias discais cervicais. A segunda situação é caracterizada pelo paciente apresentar sintomas de compressão do nervo mediano no túnel do carpo, mas concomitantemente apresenta um segundo local de compressão do nervo mediano, situação conhecida como dupla compressão. Também pode ocorrer concomitantemente à compressão do nervo mediano no túnel do carpo, a compressão do nervo ulnar, dificultando mais ainda o diagnóstico correto. A literatura descreve que os resultados do tratamento cirúrgico da STC são menos previsíveis quando existe um segundo local de compressão nervosa. ${ }^{9}$ Na terceira situação, o paciente apresenta a compressão do nervo mediano no túnel do carpo, mas possui comorbidades que também se manifestam com sintomas parestésicos como fibromialgia, diabetes mellitus, hipotireoidismo, gota, artrite reumatoide, neurites e outras doenças dos nervos periféri$\cos ^{27,28}$ Em geral os resultados pós-operatórios nos pacientes com essas comorbidades apresentam melhora dos sintomas mais lentamente que em indivíduos saudáveis, embora seja observada uma melhora significativa nos escores de gravidade dos sintomas. ${ }^{29}$ A quarta situação se caracteriza pela compressão do nervo mediano no túnel do carpo e presença de comorbidades psicológicas que afetam a percepção do paciente. Nessa situação podemos citar a presença de depressão e os interesses de ganhos secundários com o problema de saúde. ${ }^{30}$ Expectativas discordantes entre o cirurgião e o paciente com relação ao resultado final do tratamento podem levar a conflitos entre ambos. É importante o cirurgião explicar ao paciente os possíveis resultados realistas da cirurgia. Na compressão nervosa severa e de longa duração, sintomas como dormência e alterações musculares podem não se resolver completamente após a cirurgia. ${ }^{31}$

Outra situação comum na prática clínica é a ocorrência de afecções concomitantes na mão e punho em pacientes com STC. A presença prévia da artrose trapeziometacarpal, tenossinovite estenosante de De Quervain e dedo em gatilho devem ser pesquisadas, diagnosticadas e informadas porque interferem na satisfação do paciente após a descompressão cirúrgica da STC. ${ }^{29,32}$ o tratamento dessas afecções concomitantes deve ser realizado ao mesmo tempo da liberação do nervo mediano, para se evitar sintomas residuais no pósoperatório. A literatura é controversa se o tratamento 
cirúrgico da STC acelera ou predispõe o aparecimento de dedo em gatilho. ${ }^{33,34}$

Em pacientes em que temos dúvidas se eles irão se beneficiar da cirurgia, podemos utilizar a injeção de corticosteróides no interior do túnel do carpo para tentar predizer o resultado do tratamento cirúrgico. ${ }^{35}$

As causas per-operatórias mais comuns de ITCSTC são a secção incompleta do ligamento transverso do carpo (LTC), lesão nervosa intraoperatória iatrogênica e a não identificação da presença de alterações anatômicas tendinosas. Zhang et al. ${ }^{9}$ relataram que a descompressão incompleta é responsável por $50 \%$ a $58 \%$ dos pacientes com sintomas persistentes e pode provocar uma piora aguda dos sintomas devido a compressão nervosa localizada em um ponto do nervo mediano. A localização da liberação incompleta não está correlacionada com a técnica cirúrgica aberta ou endoscópica. ${ }^{12} \mathrm{Na}$ maioria dos casos, a liberação incompleta ocorre por integridade das fibras distais do LTC ou das fibras transversais proximais na prega do punho e da fáscia antebraquial distal. ${ }^{12,36}$ A lesão nervosa intraoperatória iatrogênica geralmente apresenta sintomas parestésicos constantes que não variam com a posição do punho ou com o período do dia. Karl et al. ${ }^{37}$ em estudo retrospectivo sobre ITCSTC, observaram que apenas $0,6 \%$ das complicações foram devido a lesão do nervo mediano, $0,12 \%$ foram por lesão do nervo digital, $\mathbf{0 . 0 3 \%}$ foram por lesão do nervo cutâneo palmar e $0.01 \%$ foram por lesão do ramo motor (-Fig. 1). O não reconhecimento da presença de variações anatômicas musculotendinosas são descritas como possíveis causas de compressão secundária e consequente causa da manutenção dos sintomas clínicos. ${ }^{38,39}$

As causas pós-operatórias de ITCSTC são aderências cicatriciais em torno do nervo mediano e reconstituição do ligamento transverso do carpo por tecido cicatricial. ${ }^{35}$ Tung et al. ${ }^{11}$ relataram que encontraram aderências cicatriciais em torno do nervo mediano em $88 \%$ dos seus pacientes submetidos à revisão cirúrgica. Essas aderências podem ser encontradas em todo o contorno do nervo ou localizadas, mas suficientes para impedir a excursão do nervo dentro do túnel do carpo e diminuir a vascularizaçāo do nervo. ${ }^{36}$
Reconstituição do LTC foi observada durante a cirurgia de revisão. Jones et al. ${ }^{12}$ e Mosier et al. ${ }^{40}$ relataram que a maior parte dos pacientes apresentavam menos de 1 ano de pósoperatório e durante a revisão cirúrgica encontraram o LTC reconstituído com tecido cicatricial. Uma das maneiras de evitar essas aderências em torno do nervo mediano é a utilização da via de acesso ulnar e abertura excêntrica do retináculo dos flexores na região mais ulnar, evitando dessa forma a formação de tecido cicatricial imediatamente acima do nervo mediano. ${ }^{41}$

\section{Quando revisar a cirurgia do Túnel do Carpo}

A revisão da cirurgia de descompressão do nervo mediano está indicada para os pacientes com diagnóstico de descompressão incompleta do nervo mediano, lesão iatrogênica de nervo e para casos de STC recorrente após um período prolongado de alívio dos sintomas. ${ }^{13,40}$

O'Malley et al. $^{42}$ recomendam a revisão cirúrgica em pacientes que continuam a apresentar parestesias nas mão durante a realização do teste de Phalen, despertar noturno ou sintomas parestésicos exacerbados por atividades físicas ou no trabalho. Referem que se a incisão primária foi considerada adequada e se esses sintomas não estiverem presentes, acreditam que a reexploração não resultará em um resultado satisfatório. Stang et al. ${ }^{43}$ concordam que o resultado de revisão da cirurgia é positiva, ocorrendo diminuição da dor, dos sintomas neurológicos e melhora da força, principalmente no grupo em que ocorreu recidiva dos sintomas. Em recente trabalho prospectivo, Stirling et al. ${ }^{44}$ confirmaram que os pacientes submetidos à revisão de descompressão aberta do túnel do carpo apresentaram uma melhora significativa na função e na qualidade de vida relacionada à saúde.

O insucesso da revisão cirúrgica foi relacionada a diferentes fatores. ${ }^{11}$ Cobb e Amadio ${ }^{7}$ relataram que a existência de reivindicação trabalhista por parte do paciente, presença de sintomas na distribuição do nervo ulnar e ausência de anormalidade na eletroneuromiografia pré-operatória são fatores de risco para insucesso após a revisão da cirurgia.
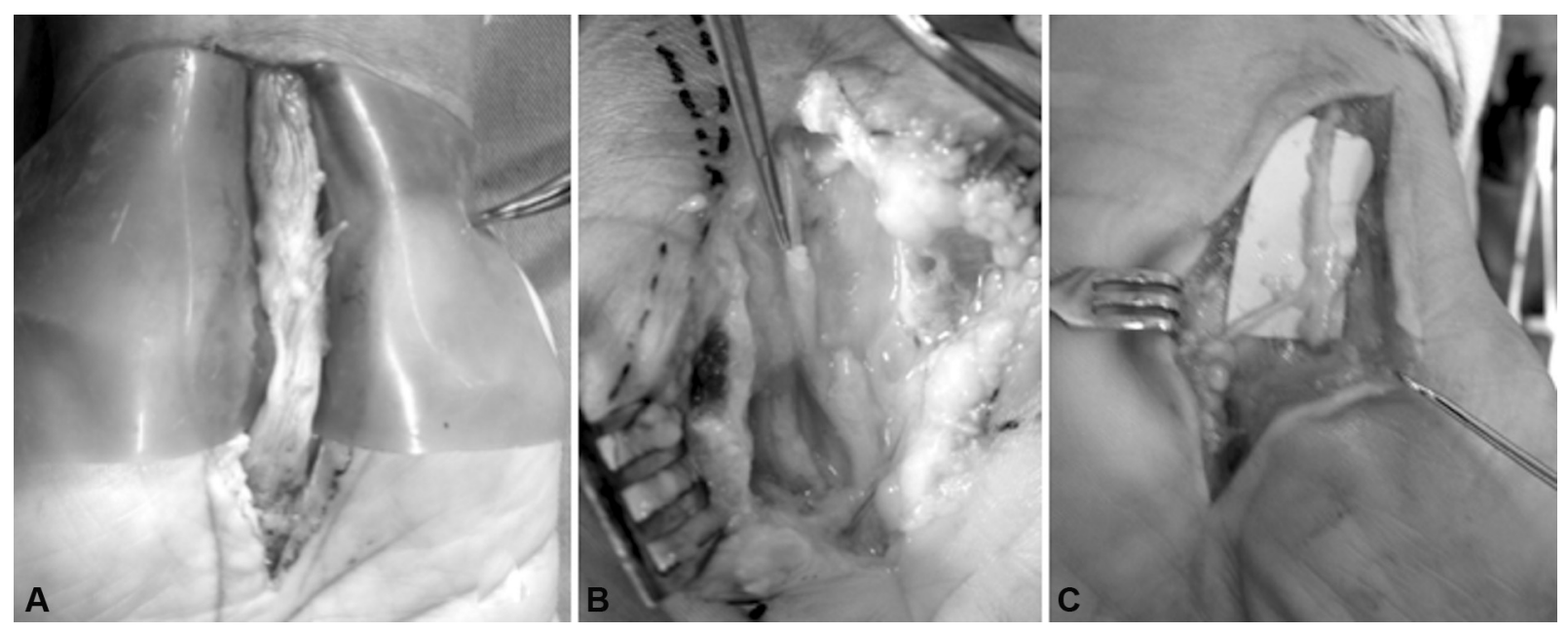

Fig. 1 Fotografia de revisões de cirurgia para descompressão do túnel do carpo demonstrando lesão iatrogênica intra-operatória do nervo. (A) Lesão do nervo mediano. (B) Lesão do nervo digital. (C) Lesão do nervo cutâneo palmar. 


\section{Como revisar a cirurgia para descompressão do Túnel do Carpo}

A revisão da cirurgia para descompressão do túnel do carpo deve ser realizada com uma abordagem aberta. Essa abordagem permitirá uma melhor observação de possíveis locais de compressão residual e presença de possíveis variações anatômicas ou aderências em torno do nervo mediano. A incisão deve iniciar de 5 a $7 \mathrm{~cm}$ proximal a prega de flexão do punho, incluir a cicatriz pré-existente e continuar em zigue-zague de Bruner para a palma da mão. ${ }^{5}$ Devido a dificuldade de dissecção pela presença de tecido cicatricial na região da cicatriz pré-existente, maior cuidado deve ser tomado para se evitar uma lesão iatrogênica do nervo mediano. Durante a avaliação do nervo, utilizando lupa ou microscópio, devemos estar atentos a regiões de compressão circunferencial tipo ampulheta, que justifique compressão recorrente ou persistente. Todo tecido que possa estar comprimindo o nervo mediano deve ser removido nessa etapa da cirurgia (-Fig. 2). Se algum local do nervo estiver lesado total ou parcialmente, deverá ser reparado com enxerto de nervo. A descompressão do nervo ulnar no canal de Guyon pode ser realizada se considerada necessária. ${ }^{5}$

Procedimentos coadjuvantes muitas vezes são necessários para restaurar o deslizamento do nervo mediano, minimizar

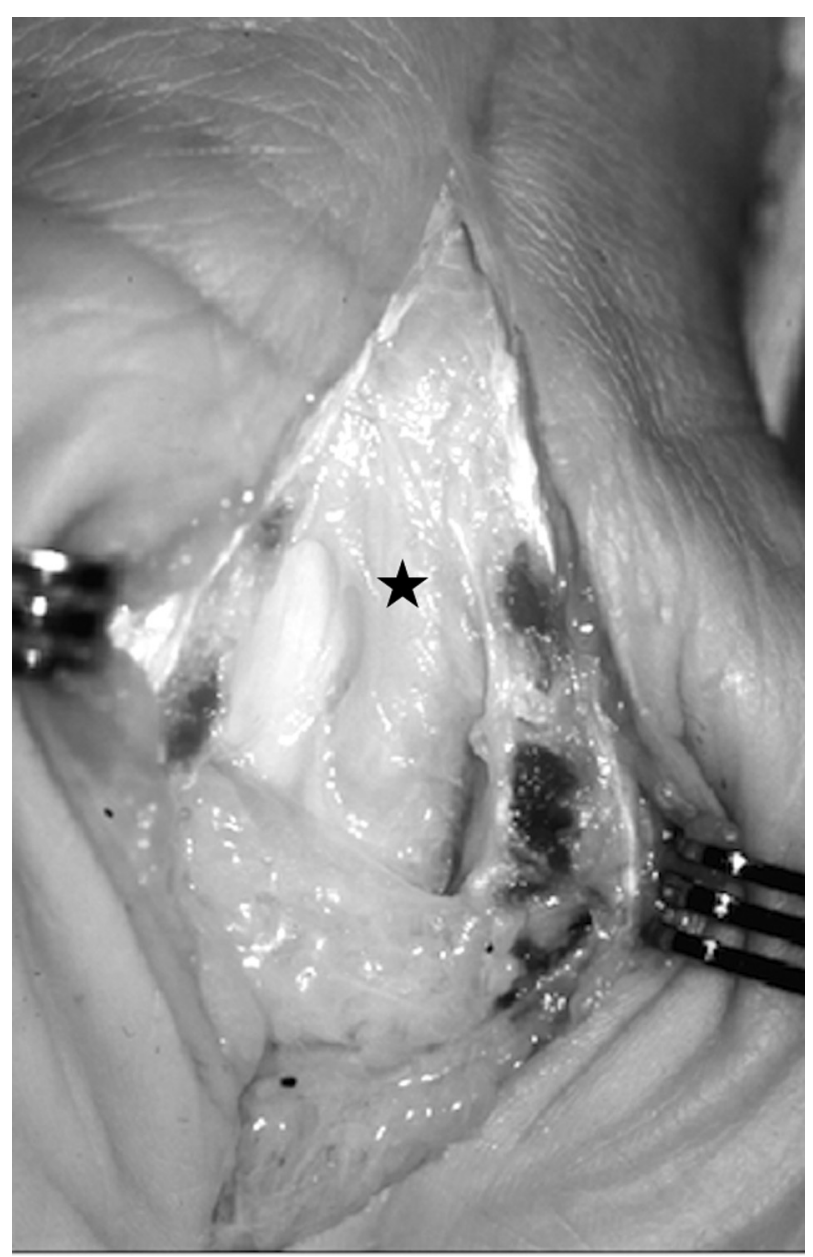

Fig. 2 Incisão ampla para permitir identificação de potenciais locais remanescentes de compressão e de aderências em torno do nervo mediano (estrela). a formação de cicatrizes e melhorar a circulação neural. ${ }^{45,46}$ Podemos realizar a cobertura do nervo com materiais sintéticos, utilizar tecido autólogo como a interposição de veia em torno do nervo mediano ou retalhos de partes moles. ${ }^{47,48}$

Materiais sintéticos podem ser utilizados para proteger o nervo mediano. São compostos de colágeno o principal componente da matrix extracelular. A vantagem desses materiais é a não necessidade de incisões adicionais, porém ainda não existem evidências clínicas de sua superioridade em relação aos outros métodos. ${ }^{49}$

O conceito de interposição de veia, colocando a veia em torno do nervo mediano como barreira para prevenir formação de cicatriz, foi originalmente descrito em ratos mas tem sido utilizada clinicamente. ${ }^{48,50} \mathrm{~A}$ técnica cirúrgica é composta pela liberação do nervo mediano das aderências cicatriciais e identificação da região que deverá ser coberta por veia. Após a retirada de 25 a $30 \mathrm{~cm}$ de veia safena, esta é aberta longitudinalmente. Com a porção da íntima em contato com o nervo mediano, a veia é enrolada no nervo e a cada 360 graus é realizado um ponto com fio 8-0 para manter a veia no local. Varitimidis et al. ${ }^{50}$ em estudo clínico observaram diminuição da dor e sintomas parestésicos, melhora da sensibilidade avaliada pela discriminação de dois pontos e melhora nos parâmetros da eletroneuromiografia. Consideramos como desvantagem dessa técnica, a necessidade da incisão adicional necessária para retirada do enxerto.

Diferentes retalhos tem sido descritos para proteger o nervo mediano e melhorar a circulação local. $\mathrm{O}$ retalho adiposo da eminência hipotenar foi popularizado por Strickland $^{51}$ (-Fig. 3). Após a descompressão do nervo mediano e

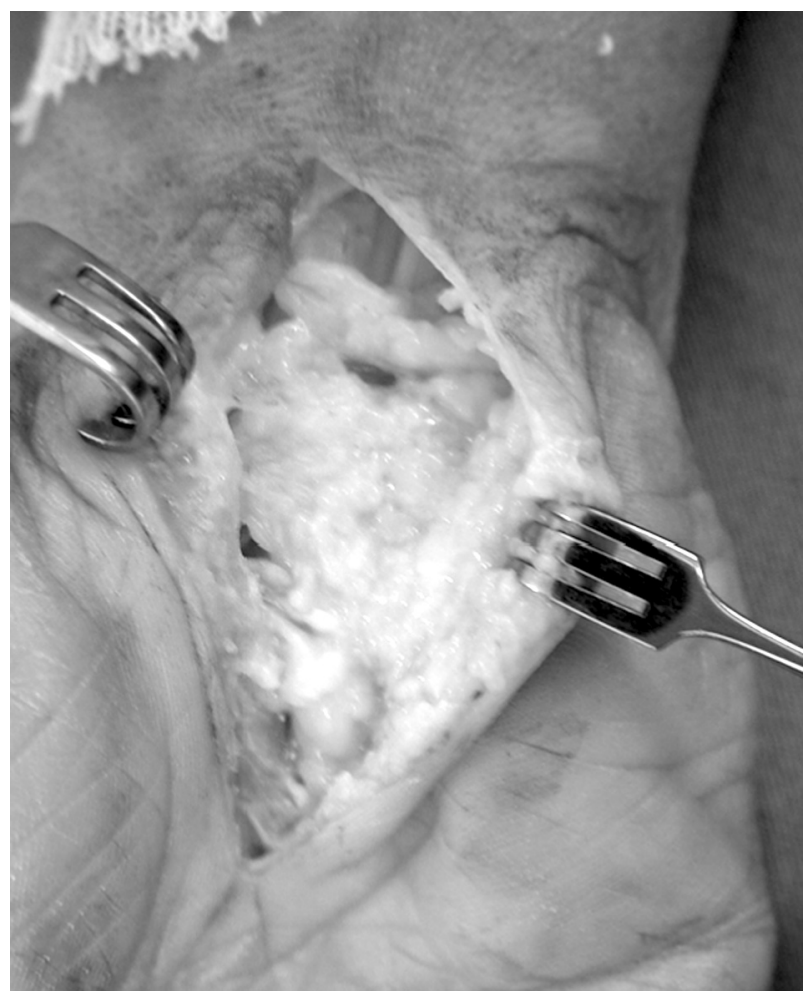

Fig. 3 Um retalho de tecido adiposo da eminência hipotenar foi realizado para interpor tecido adiposo entre o nervo mediano e o ligamento transverso do carpo transversal e a cicatriz cirúrgica. 

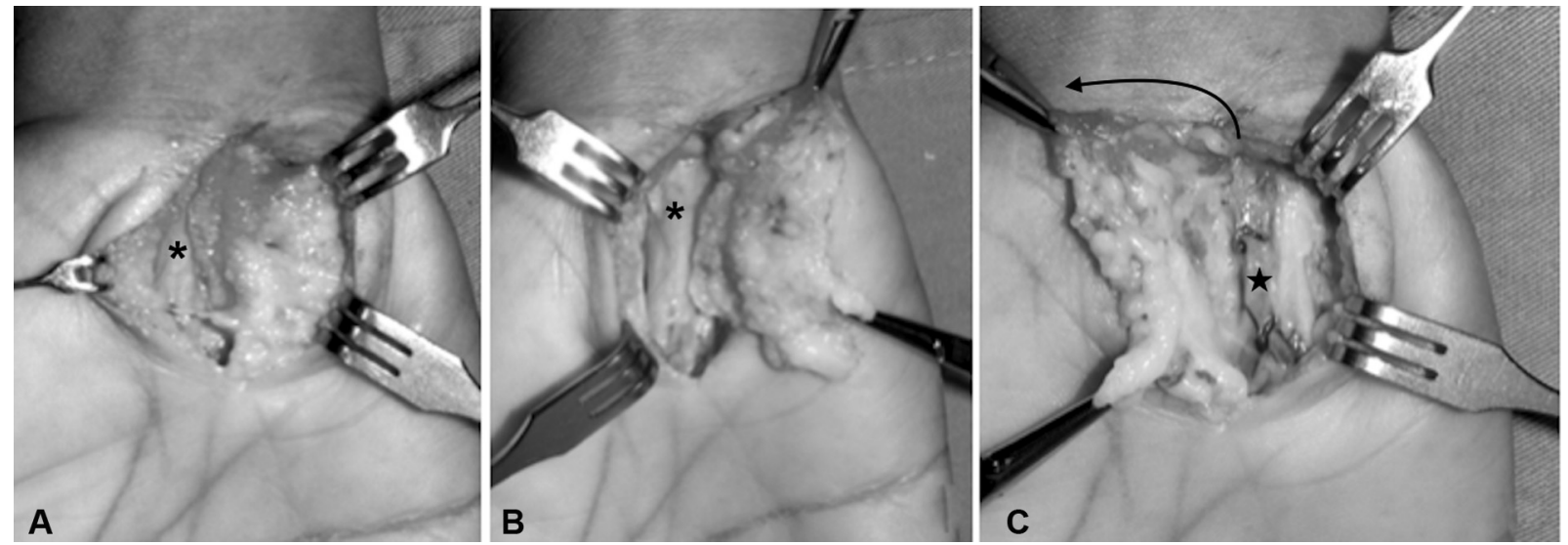

Fig. 4 Após a remoção do tecido que pode comprimir o nervo mediano $\left({ }^{*}\right)$, o tecido adiposo é dissecado até que os músculos hipotenares e o músculo palmar curto sejam identificados (A). Como o retalho é irrigado pela artéria ulnar(estrela), ele é elevado (B), girado 180 graus (seta preta) e transposto na direção radial para cobrir o nervo mediano (C).

retirada das aderências, realiza-se a confecção do retalho de tecido adiposo que é interposto entre o nervo mediano e o LTC. $^{51,52}$ Silva et al..$^{52}$ utilizando esse retalho, obtiveram melhora dos sintomas em 13 de 15 pacientes submetidos a cirurgia por essa técnica. Zummioti et al. ${ }^{45}$ obtiveram resultados considerados bons em 15 pacientes. Fusetti et al. ${ }^{46}$ tiveram resolução dos sintomas após 6 meses de cirurgia em 18 dos 20 pacientes tratados com esse retalho. 0 retalho de gordura hipotenar também pode ser realizado na chamada forma reversa, onde o retalho é rodado 180 graus para cobrir o canal do $\operatorname{carpo}^{53}$ (-Fig. 4).

O retalho fascial da artéria radial foi utilizado em seis pacientes com bons resultados, mas tem a desvantagem de sacrificar a artéria radial. ${ }^{54} \mathrm{O}$ retalho lateral dorsal do antebraço foi utilizado com bons resultados tendo a vantagem de se preservar a artéria radial. ${ }^{55}$

Vogelin et al..$^{56}$ utilizaram o retalho da artéria interóssea posterior e com seguimento médio de 2 anos, 12 dos 14 pacientes tiveram melhora significativa da dor.

Entre as opções de tratamento retalhos musculares foram descritos, mas apresentam a desvantagem que a quantidade de tecido fornecido pode ser inadequado para cobrir toda a extensão da região. ${ }^{57}$

Os retalhos livres são tecnicamente mais trabalhosos e devem ser utilizados como último recurso, em pacientes que já foram submetidos a vários procedimentos anteriores, incluindo retalhos locais. ${ }^{57}$

Uma meta-análise sistemática comparou os resultados da revisão cirúrgica simples com a revisão associada a confecção de retalho para proteção do nervo mediano. Noventa e quatro pacientes de 14 estudos tratados com cobertura de retalho foram comparados com 364 pacientes de 9 estudos que usaram descompressão aberta e neurólise isoladamente. 0 grupo com retalho teve taxa de sucesso de $86 \%$ em comparação com $75 \%$ no grupo sem retalho $(\mathrm{P}=0,001)$. Apesar da maior taxa de sucesso, o grupo com retalho apresentou maior taxa de complicações como sintomas de parestesias, sensibilidade na cicatriz e complicações da ferida. ${ }^{58}$

\section{Considerações Finais}

O ITCSTC pode ocorrer em um significativo número de pacientes que foram submetidos a liberação do túnel do carpo. Nossa recomendação nesses casos é iniciar com uma nova avaliação clínica para descartar diagnósticos diferenciais. Caso confirmado que o problema é local, realizar uma incisão ampliada que inclua a cicatriz cirúrgica pre-existente, realizar uma liberação do nervo mediano de qualquer compressão ou cicatriz em torno do nervo mediano. Se houver cicatrizes significativas ou fibrose perineural, um retalho deve ser realizado para diminuir a formação de novas aderências e fornecer interposição com neovascularização.

\section{Conflito de Interesses}

Os autores declaram não haver conflito de interesses.

\section{Referências}

1 Atroshi I, Gummesson C, Johnsson R, Ornstein E, Ranstam J, Rosén I. Prevalence of carpal tunnel syndrome in a general population. JAMA 1999;282(02):153-158

2 Karpitskaya Y, Novak CB, Mackinnon SE. Prevalence of smoking, obesity, diabetes mellitus, and thyroid disease in patients with carpal tunnel syndrome. Ann Plast Surg 2002;48(03): 269-273

3 Pourmemari MH, Heliövaara M, Viikari-Juntura E, Shiri R. Carpal tunnel release: Lifetime prevalence, annual incidence, and risk factors. Muscle Nerve 2018;58(04):497-502

4 Verdugo RJ, Salinas RA, Castillo JL, Cea JG. Surgical versus nonsurgical treatment for carpal tunnel syndrome. Cochrane Database Syst Rev 2008;(04):CD001552

5 Lauder A, Mithani S, Leversedge FJ. Management of recalcitrant carpal tunnel syndrome. J Am Acad Orthop Surg 2019;27(15):551-562

6 Louie D, Earp B, Blazar P. Long-term outcomes of carpal tunnel release: a critical review of the literature. Hand (N Y) 2012;7(03):242-246

7 Cobb TK, Amadio PC. Reoperation for carpal tunnel syndrome. Hand Clin 1996;12(02):313-323

8 Gil JA, Bokshan S, Genovese T, Got C, Daniels AH. Medical malpractice following carpal tunnel surgery. Orthopedics 2018; 41(04):e569-e571

9 Zhang D, Earp BE, Blazar P. Evaluation and Management of Unsuccessful Carpal Tunnel Release.J Hand Surg Am 2019;44(09):779-786 
10 Zieske L, Ebersole GC, Davidge K, Fox I, Mackinnon SE. Revision carpal tunnel surgery: a 10-year review of intraoperative findings and outcomes. J Hand Surg Am 2013;38(08):1530-1539

11 Tung TH, Mackinnon SE. Secondary carpal tunnel surgery. Plast Reconstr Surg 2001;107(07):1830-1843, quiz 1844, 1933

12 Jones NF, Ahn HC, Eo S. Revision surgery for persistent and recurrent carpal tunnel syndrome and for failed carpal tunnel release. Plast Reconstr Surg 2012;129(03):683-692

13 Beck JD, Brothers JG, Maloney PJ, Deegan JH, Tang X, Klena JC. Predicting the outcome of revision carpal tunnel release. J Hand Surg Am 2012;37(02):282-287

14 Keith MW, Masear V, Chung KC, et al. American Academy of Orthopaedic Surgeons Clinical Practice Guideline on diagnosis of carpal tunnel syndrome. J Bone Joint Surg Am 2009;91(10):2478-2479

15 Elfar JC, Calfee RP, Stern PJ. Topographical assessment of symptom resolution following open carpal tunnel release. J Hand Surg Am 2009;34(07):1188-1192

16 Cheng CJ, Mackinnon-Patterson B, Beck JL, Mackinnon SE. Scratch collapse test for evaluation of carpal and cubital tunnel syndrome. J Hand Surg Am 2008;33(09):1518-1524

17 Dawson DM. Entrapment neuropathies of the upper extremities. N Engl J Med 1993;329(27):2013-2018

18 Dellon AL, Mackinnon SE, Brandt KE. The markings of the Semmes-Weinstein nylon monofilaments. J Hand Surg Am 1993;18 (04):756-757

19 Lehman LF, Orsini MB, Nicholl AR. The development and adaptation of the Semmes-Weinstein monofilaments in Brazil. J Hand Ther 1993;6(04):290-297

20 Chammas M, Boretto J, Burmann LM, Ramos RM, Dos Santos Neto FC, Silva JB. Carpal tunnel syndrome - Part I (anatomy, physiology, etiology and diagnosis). Rev Bras Ortop 2014;49 (05):429-436

21 Fowler JR, Byrne K, Pan T, Goitz RJ. False-positive rates for nerve conduction studies and ultrasound in patients without clinical signs and symptoms of carpal tunnel syndrome. J Hand Surg Am 2019;44(03):181-185

22 Fowler JR, Munsch M, Huang Y, Hagberg WC, Imbriglia JE. Preoperative electrodiagnostic testing predicts time to resolution of symptoms after carpal tunnel release. J Hand Surg Eur Vol 2016; 41(02):137-142

23 Pimentel BF, Abicalaf CA, Braga L, et al. Cross-sectional area of the median nerve characterized by Ultrasound in Patients With Carpal Tunnel Syndrome Before and After the Release of the Transverse Carpal Ligament. J Diagn Med Sonogr 2013;29(03): 116-121

24 de Campos CC, Manzano GM, de Andrade LB, Castelo Filho A, Nóbrega JAM. Tradução e validação do questionário de avaliação de gravidade dos sintomas e do estado funcional na síndrome do tú nel do carpo. Arq Neuropsiquiatr 2003;61(01):51-55

25 Matsuo RP, Fernandes CH, Meirelles LM, Raduan Neto J, Dos Santos JBG, Fallopa F. Translation and cross - cultural adaptation of the 6item carpal tunnel syndrome symtoms scale and palmar pain scale questionnaire into brazilian Portuguese. Hand (N Y) 2016;11 (02):168-172

26 Fernandes CH, Neto JR, Meirelles LM, Pereira CN, Dos Santos JB, Faloppa F. Translation and cultural adaptation of the Brief Michigan Hand Questionnaire to Brazilian Portuguese language. Hand (N Y) 2014;9(03):370-374

27 Eroğlu A, Sarı E, Topuz AK, Şimşek H, Pusat S. Recurrent carpal tunnel syndrome: Evaluation and treatment of the possible causes. World J Clin Cases 2018;6(10):365-372

28 Straub TA. Endoscopic carpal tunnel release: a prospective analysis of factors associated with unsatisfactory results. Arthroscopy 1999;15(03):269-274

29 Kim JH, Gong HS, Lee HJ, Lee YH, Rhee SH, Baek GH. Pre- and postoperative comorbidities in idiopathic carpal tunnel syndrome: cervical arthritis, basal joint arthritis of the thumb, and trigger digit. J Hand Surg Eur Vol 2013;38(01):50-56
30 Lozano Calderón SA, Paiva A, Ring D. Patient satisfaction after open carpal tunnel release correlates with depression.J Hand Surg Am 2008;33(03):303-307

31 Kamiya H, Kimura M, Hoshino S, Kobayashi M, Sonoo M. Prognosis of severe carpal tunnel syndrome with absent compound muscle action potential. Muscle Nerve 2016;54(03):427-431

32 Joshy S, Thomas B, Ghosh S, Haidar SG, Deshmukh SC. Patient satisfaction following carpal-tunnel decompression: a comparison of patients with and without osteoarthritis of the wrist. Int Orthop 2007;31(01):1-3

33 Harada K, Nakashima H, Teramoto K, Nagai T, Hoshino S, Yonemitsu H. Trigger digits-associated carpal tunnel syndrome: relationship between carpal tunnel release and trigger digits. Hand Surg 2005;10(2-3):205-208

34 Gancarczyk SM, Strauch RJ. Carpal tunnel syndrome and trigger digit: common diagnoses that occur "hand in hand". J Hand Surg Am 2013;38(08):1635-1637

35 Green DP. Diagnostic and therapeutic value of carpal tunnel injection. J Hand Surg Am 1984;9(06):850-854

36 Stutz N, Gohritz A, van Schoonhoven J, Lanz U. Revision surgery after carpal tunnel release-analysis of the pathology in 200 cases during a 2 year period. J Hand Surg Am 2006;31 (01):68-71

37 Karl JW, Gancarczyk SM, Strauch RJ. Complications of carpal tunnel release. Orthop Clin North Am 2016;47(02):425-433

38 Mimura T, Uchiyama S, Hayashi M, Uemura K, Moriya H, Kato H. Flexor carpi radialis brevis muscle: A case report and its prevalence in patients with carpal tunnel syndrome. J Orthop Sci 2017; 22(06):1026-1030

39 Keese GR, Wongworawat MD, Frykman G. The clinical significance of the palmaris longus tendon in the pathophysiology of carpal tunnel syndrome. J Hand Surg [Br] 2006;31(06):657-660

40 Mosier BA, Hughes TB. Recurrent carpal tunnel syndrome. Hand Clin 2013;29(03):427-434

41 Galbiatti JA, Komatsu S, Faloppa F, Albertoni WM, Silva SE. Via de acesso ulnal na síndrome do túnel do carpo -. Rev Bras Ortop 1991;26(11/12):389-394

42 O'Malley MJ, Evanoff M, Terrono AL, Millender LH. Factors that determine reexploration treatment of carpal tunnel syndrome. J Hand Surg Am 1992;17(04):638-641

43 Stang F, Stütz N, Lanz U, van Schoonhoven J, Prommersberger KJ. [Results after revision surgery for carpal tunnel release]. Handchir Mikrochir Plast Chir 2008;40(05):289-293

44 Stirling PHC, Yeoman TFM, Duckworth AD, Clement ND, Jenkins PJ, McEachan JE. Decompression for recurrent carpal tunnel syndrome provides significant functional improvement and patient satisfaction. J Hand Surg Eur Vol 2020;45(03):250-254

45 Zumiotti AV, Ohno PE, Prada FS, Azze RJ. Complicações do tratamento cirúrgico da síndrome do túnel do carpo. Rev Bras Ortop 1996;31(03):199-202

46 Fusetti C, Garavaglia G, Mathoulin C, Petri JG, Lucchina S. A reliable and simple solution for recalcitrant carpal tunnel syndrome: the hypothenar fat pad flap. Am J Orthop 2009;38(04): 181-186

47 Chamas M, Boretto J, Burmann LM, Ramos RM, Santos Neto FC, Silva JB. Syndrome do tonel do Carpo- Parte II (Tratamento). Rev Bras Ortop 2014;49(05):437-445

$48 \mathrm{Xu} \mathrm{J}$, Sotereanos DG, Moller AR, et al. Nerve wrapping with vein grafts in a rat model: a safe technique for the treatment of recurrent chronic compressive neuropathy. J Reconstr Microsurg 1998;14(05):323-328, discussion 329-330

49 Dy CJ, Aunins B, Brogan DM. Barriers to epineural scarring: Role in treatment of Traumatic nerve injury and chronic compressive neuropathy. J Hand Surg Am 2018;43(04):360-367

50 Varitimidis SE, Vardakas DG, Goebel F, Sotereanos DG. Treatment of recurrent compressive neuropathy of peripheral nerves in the upper extremity with an autologous vein insulator. J Hand Surg Am 2001;26(02):296-302 
51 Strickland JW, Idler RS, Lourie GM, Plancher KD. The hypothenar fat pad flap for management of recalcitrant carpal tunnel syndrome. J Hand Surg Am 1996;21(05):840-848

52 Silva JB, Fontes Neto P, Rio JT, Fridman M. Retalho hipotenarianoadiposo na recidiva da síndrome do túnel do carpo. Rev Bras Ortop 1996;31(12):1019-1022

53 Ramos RFM, Meneguzzi K, Pellicioli A, Varela G, Calcagnotto FN, Silva JB. Retalho tenar adipofascial reverso para cobertura do nervo mediano hipertrofiado em macrodactilia. Rev Bras Cir Plást 2015;30(04):674-679

54 Tham SK, Ireland DC, Riccio M, Morrison WA. Reverse radial artery fascial flap: a treatment for the chronically scarred median nerve in recurrent carpal tunnel syndrome. J Hand Surg Am 1996; 21(05):849-854
55 Galbiatti JA, Gilbert A, Brunelli F, et al. Study of anatomical bases of a latero-dorsal flap of the forearm preserving the radial artery. Rev Paul Med 1992;110(01):14-19

56 Vögelin E, Bignion D, Constantinescu M, Büchler U. [Revision surgery after carpal tunnel release using a posterior interosseous artery island flap]. Handchir Mikrochir Plast Chir 2008;40(02): $122-127$

57 Abzug JM, Jacoby SM, Osterman AL. Surgical options for recalcitrant carpal tunnel syndrome with perineural fibrosis. Hand (N Y) 2012;7(01):23-29

58 Soltani AM, Allan BJ, Best MJ, Mir HS, Panthaki ZJ. A systematic review of the literature on the outcomes of treatment for recurrent and persistent carpal tunnel syndrome. Plast Reconstr Surg 2013;132(01):114-121 